\title{
Las organizaciones internacionales y sus recursos de poder. Una propuesta analítica*
}

\author{
Germán Burgos Silva (Colombia)**
}

\section{Resumen}

En el texto se responde la pregunta sobre cuáles pueden considerarse recursos de poder de algunas organizaciones internacionales $(\mathrm{Ol})$, en cuanto entes relativamente autónomos de los Estados que las crean. Se sostiene que, según sea el caso, la producción de cierta normativa, la información, la coerción y el componente simbólico son parte de dichos mecanismos, aunque existen solapamientos entre ellos que dificultan su distinción. El análisis se funda en una perspectiva sociopolítica de las OI, con la que se busca complementar la mirada predominantemente jurídica de estas. Por lo tanto, va más allá de visualizar aquellas como meros instrumentos de los Estados y que tienen apenas poderes delegados por estos, estableciendo la existencia de ámbitos que permiten identificar espacios de autonomía y de poder sobre los Estados.

\section{Palabras clave}

Política Internacional; Instituciones Políticas; Teoría Política; Organizaciones Internacionales; Poder.

Fecha de recepción: agosto de 2018 • Fecha de aprobación: noviembre de 2018

\section{Cómo citar este artículo}

Burgos Silva, Germán. (2019). Las organizaciones internacionales y sus recursos de poder. Una propuesta analítica. Estudios Políticos (Universidad de Antioquia), 54, pp. 149-176. http://doi.org/10.17533/udea.espo.n54a07

\footnotetext{
*Artículo de reflexión producto de la investigación OI y responsabilidad internacional, en el marco del grupo A de Colciencias Estado, derecho y territorio de la Universidad Libre, Colombia.

** Abogado. Especialista en Ciencia Política. Doctor en Derecho. Profesor asociado de la Universidad Nacional de Colombia. Profesor del doctorado en Derecho de la Universidad Libre, Colombia. Correo electrónico: jgburgoss@unal.edu.co Orcid: https://orcid.org/0000-0001-9361-990X
} 


\title{
International Organizations and their Power Resources. An Analytical Proposal
}

\begin{abstract}
This study set out to answer the question of what can be considered to be the power resources of international organizations (IOs), as entities partially autonomous from the States that create them. It is argued that the production of certain regulations, the production of information, coercion, and the symbolic component are part of these resources. Thus, the existing literature is reviewed, while attempting to recognize the overlaps and limitations of these resources. The analysis is based on a sociopolitical vision of IOs that seeks to complement the dominant legal view of them; therefore, it goes beyond viewing IOs as mere instruments of States with delegated powers. The study establishes the existence of scopes that allow for the identification of spaces of autonomy and, as a consequence, of power over States by this kind of organizations.
\end{abstract}

\section{Keywords}

International Policy; Political Institutions; Political Theory; International Organizations; Power. 


\section{Introducción}

De acuerdo con la prensa internacional, soldados franceses, integrantes de las Fuerzas de Paz aprobadas por la Organización de las Naciones Unidas (ONU), sometieron a niños de la República Centroafricana a vejaciones sexuales a cambio de comida. Dicha situación se conoció en el interior de esta organización gracias a un informe oficial al respecto, el cual no tuvo mayor impacto, salvo cuando un funcionario de la entidad lo filtró al Estado francés. Como parte de la respuesta, la ONU abrió una investigación disciplinaria contra el burócrata que reveló parcialmente dicha información, aunque también creó un panel independiente para analizar las denuncias (Botero, 2016, julio 7).

En un terreno menos extremo, a organizaciones internacionales (OI) como el Banco Mundial (BM) y el Fondo Monetario Internacional (FMI) las han cuestionado por el impacto de sus acciones en materia de derechos económicos y sociales, en tanto que a la Organización Mundial de la Salud (OMS) la critican por su política de promoción de las vacunas; por su parte, el movimiento social de mujeres ha logrado que la ONU se comprometa más con sus reivindicaciones, el Mercado Común del Sur (Mercosur) amplía su espectro con la inclusión de nuevos países y, a su vez, la Organización Mundial del Comercio $(\mathrm{OMC})$ afronta una crisis como producto de la generación de tratados bilaterales de libre comercio e inversión que le quitan centralidad (Brodnig, 2001; Wilkinson, 2013; Dreyzin y Morales, 2009; Women UN, 2015).

En medio de los roles y alcances que tienen organizaciones como las ya nombradas, lo que podemos visualizar en común frente a situaciones como las anteriores es el protagonismo creciente y estratégico que las $\mathrm{OI}$ en general han venido adquiriendo en el escenario internacional, de manera tal que hoy es fácil constatar que superan numéricamente a los Estados; es más, algunas de ellas hacen tareas que antes realizaban aquellos, afectando por tanto la vida de las personas y demás actores; asimismo, los individuos y grupos sociales buscan internacionalizar su lucha a través de estas plataformas, en tanto que varios agentes internacionales formales e informales dependen cada vez más de la información y la acción técnica de las OI.

Ahora bien, reconocer que estos organismos tienen atribuciones variadas y que afectan la vida de varios actores hace referencia, en el fondo, al estudio del poder de las OI, o lo que en otros términos podría verse 
como la identificación de cuáles pueden ser sus recursos para lograr que los obedezcan.

El abordaje de las OI como actores de poder se ha planteado de diversas maneras. Desde el punto de vista del derecho internacional, se ha sostenido que estos sujetos son ante todo entes derivados de la voluntad de los Estados, y que para juzgarlos como tales precisan de la dotación de una personalidad jurídica propia, que les permita tener derechos y obligaciones (Ridruejo, 2012). Si bien por esta vía se presume que las Ol son dependientes en forma sustancial de sus creadores, la discusión sobre los llamados poderes implícitos e inherentes ha permitido admitir que estas tienen un margen de acción propio, a partir de la asunción de facultades que los Estados no les han dado explícitamente y que, por tanto, pueden considerarse como actores de poder por sí mismos y parcialmente independientes de los Estados.

Por otra parte, desde una lectura más política, se dice que algunas OI son instrumentos de los Estados centrales o potencias, que las utilizan como mecanismos para enmascarar y transmitir sus agendas particulares en el marco de las luchas y diferencias de poder en los ámbitos geopolítico y geoeconómico (Foot, MacFarlane y Mastanduno, 2003). Se sostiene entonces,

[152] por ejemplo, que el FMI y el Banco Mundial son meros valedores de las agendas de Europa Occidental y Estados Unidos, con la lógica de mantener un orden económico mundial que los favorece y en contra del llamado Sur o periferia. En una lógica más estructural, autores como Juan Ramón Capella (1997) y Antonio Negri (2005) han sostenido que ciertas Ol forman parte del Imperio o de un «soberano supraestatal difuso» que agencia y hace viable la globalización del neoliberalismo.

A partir de estos y otros enfoques el debate que se plantea versa sobre hasta qué punto las OI son entes más o menos autónomos de los Estados, es decir, si son meros instrumentos vacíos en manos de aquellos e incluso bajo el control de los más poderosos, o si, por el contrario, poseen márgenes de independencia que les permiten tener una vida propia, pero sobre todo ser actores de poder sobre los Estados y otros actores. Con argumentos tanto jurídicos como de la teoría de las organizaciones y de las relaciones internacionales se puede plantear que las OI tienen un nivel de autonomía variable en relación con los Estados. A partir de esto se debe admitir que dichos organismos pueden estimarse como actores de poder en cuanto tales y que, por consiguiente, entran en relaciones con varios actores. 
Con este artículo se procura identificar los recursos de poder que algunas Ol tienen para pretender y lograr cierto tipo de obediencia, especialmente respecto de los Estados-nación. Por lo tanto, ¿cuáles serían los recursos de poder con que cuentan ciertas $\mathrm{OI}$, que les permiten un nivel de influencia o de obtención de obediencia por parte de los Estados u otros actores de poder? Como respuesta a esta inquietud e hipótesis, se plantea desde un enfoque sociopolítico del estudio del poder que las Ol cuentan con recursos normativo, informativo, coercitivo o simbólico que se pueden traslapar en diversos momentos.

El alcance analítico de esta propuesta depende, hasta cierto punto, de las OI existentes, de modo tal que en algunos casos la consideración del tipo de recursos aquí expuestos cabrá plenamente, mientras en otros no será aplicable; por tanto, este trabajo tiene una dimensión parcialmente exploratoria y asociada a las particularidades del abigarrado mundo de las OI.

\section{Las organizaciones internacionales como actores «autónomos» de los Estados}

En este apartado se pretende identificar y desarrollar varios argumentos que permitan sostener que las $\mathrm{OI}$, a pesar de los enfoques realistas, cuentan con un nivel de autonomía respecto de los Estados que las crean, de modo que pueden pasar de ser meramente sus instrumentos, a lograr que aquellos las obedezcan.

\subsection{Los poderes jurídicos de las organizaciones internacionales}

Las OI son, desde el punto de vista del derecho internacional, sujetos de este y resultado de la creación generalmente de Estados por medio de tratados internacionales, dotadas de personalidad jurídica de manera directa o indirecta, lo cual les permite el cumplimiento de fines más o menos específicos, según sea el caso. Dentro de la terminología de esta disciplina, las OI son sujetos derivados, es decir, producto de decisiones estatales, y en tal sentido se asume de una u otra manera que su existencia y su funcionamiento descansan en los actores que las originan (Ridruejo, 2012). Con base en esto se ha construido la teoría de los poderes de las Ol como atribuciones de los Estados. 
Es así como se ha sostenido que los poderes de las OI pueden identificarse como implícitos, inherentes y atribuidos. Estos últimos son aquellos que sus creadores han entregado expresamente a la organización y que, por tanto, son relativamente claros y determinables. Los implícitos son aquellos necesarios para cumplir con los fines de la organización y, como puede deducirse, no son del todo claros, además ser el producto de la forma como la organización y sus mecanismos interpretan su mandato. Por su parte, los inherentes son aquellos que no están expresamente prohibidos al organismo y que, por tanto, se puede deducir que los tiene. Esta sería una clara diferenciación con la idea propia del Estado de derecho, según la cual los poderes del Estado son solo aquellos facultados expresamente por normas previas, al menos de manera ideal (Urueña, 2008, p. 244; Ku, 2000; Querejazu, 2013). Los poderes no expresos se pueden ejemplificar en la condicionalidad de los préstamos del Banco Mundial o del FMI, o en la capacidad del Consejo de Seguridad de la ONU de definir el contenido de la noción de intervención humanitaria. Con todo, al no ser prohibiciones expresas en las cartas constitutivas de dichos organismos, pueden conllevar el solapamiento entre los poderes implícitos y los inherentes, lo cual haría la diferenciación poco clara.

En otra vertiente analítica, Jean d'Aspremont (2007) plantea que los [154] Estados delegan poderes a las Ol al menos de tres formas: en primer lugar, está la relación de agencia, en la que aquellos entregan ciertas atribuciones al organismo, pero manteniendo cierto control y capacidad de decisión sobre este; en segundo término, los poderes delegados, que corresponden a eventos en los que los Estados no ejercen control sobre lo que hace o deja de hacer la Ol como producto de dicha delegación; finalmente, la transferencia de poderes, la cual no solo es difícil de controlar sino que conlleva la entrega de atribuciones del Estado a una organización que la ejerce en forma exclusiva, es decir, monopólica. Un ejemplo de esto último puede ser el Banco Central Europeo (BCE), en cuanto parte de la Unión Europea (UE), y su facultad de emitir el euro.

Dos ideas saltan a la vista del breve repaso anterior. Por un lado, en un sentido formal, parece claro que los poderes de las OI van más allá de las expresas atribuciones entregadas por los Estados e incluyen aquellas otras que se consideran — por lo general, en forma interna y sin mayor controlpoderes inherentes o implícitos (Urueña, 2008, p. 245). En otras palabras, se tendría que admitir — aun dentro de la discusión estrictamente jurídica- que los poderes de las OI sobre los Estados van más allá de aquellos entregados 
oficialmente por estos. Por el otro, más allá de la lectura jurídica, existe un creciente debate analítico sobre cómo caracterizar los ámbitos de poder que tienen dichos organismos, lo cual entraña necesariamente abordar cuál ha sido el análisis sobre la materialidad real de tales atribuciones.

\subsection{Las organizaciones internacionales desde algunos enfoques de las relaciones internacionales}

Desde el realismo, centrado en presumir que los Estados son el eje de las relaciones internacionales y actúan en forma egoísta, las Ol desempeñan dos roles: por un lado, son meros foros más o menos vacíos en los que los actores estatales están a la expectativa para llegar a acuerdos o no, al margen de algún papel cumplido por las organizaciones; por el otro, en una línea más neorrealista, se admite que las OI tienen un margen de acción propio, el cual está determinado por los intereses de las potencias mundiales, que son las que realmente deciden los aspectos fundamentales respecto del accionar de la organización (Urueña, 2008, p. 240; Carlsnaes, Risse y Simmons, 2002).

La primera y global entrada nos plantea que las OI se han creado en clave de los intereses de los Estados hegemónicos y como parte de sus estrategias para imponer y encubrir sus agendas, sobre todo en los ámbitos económico y político (Wade, 2001; 2002). En esta coyuntura estarían el Banco Mundial, el FMI o la OEA, que se visualizan como los mejores valedores de los intereses de Estados Unidos o, en el mejor de los casos, conforman parte del «imperio» del neoliberalismo o son un soberano supraestatal difuso (Capella, 1997; Negri, 2005).

Los argumentos que sostienen esta tesis están relacionados con la estructura de los órganos de decisión y el manejo de las fuentes financieras de estas $\mathrm{OI}$, los cuales estarían controlados por ciertas potencias y por el análisis de parte de su agenda, que se visualiza como funcional a los intereses del hegemón (Stone, 2013). Además, se afirma que a las potencias les conviene la existencia de las $\mathrm{Ol}$, pues pueden tramitar sus diferencias de una manera más ágil y menos costosa y, de paso, encubrir legitimadoramente sus intereses más egoístas.

Lo que no responde esta línea analítica es cómo entender que una creciente cantidad de Ol terminan asumiendo atribuciones de Estado y poniéndose de algún modo por encima de estos, logrando ámbitos de influencia y obediencia. En esta lectura tampoco se considera la dinámica 
interna de los organismos y la dotación que su personalidad jurídica puede producir en cuanto a la generación de unos ámbitos propios de acción de la OI, más allá de lo estrictamente administrativo.

En una respuesta parcial a lo anterior, con un enfoque liberal, se asume críticamente que el propósito de los Estados no es perseguir sus intereses, sino que son un medio para hacer viables las expectativas de otros actores o preocupaciones comunes tales como los individuos, los pueblos o el mismo medio ambiente. A partir de estos se conforma una agenda de lo público internacional, en la que la finalidad de las Ol es posibilitar, entre otros, mayores grados de libertad y bienestar individual, espacios de autodeterminación colectiva o derechos como un desarrollo sostenible. Esta acción colectiva, mediante dichos entramados, es también el producto de la interdependencia de aquellos países que no pueden afrontar en forma aislada ciertas situaciones, por lo que han de asumirse colectivamente a partir de las OI (Urueña, 2008, p. 85). Uno de los aportes de fondo de esta perspectiva consiste en admitir que las $\mathrm{OI}$ tienen vida propia y, por consiguiente, son de alguna forma autónomas con respecto a los Estados.

\subsection{La «autonomía» de las organizaciones internacionales: los argumentos}

Parece claro que buena parte de las OI son creación de los Estados y que en varios casos controlan sus presupuestos, seleccionan sus directivos y establecen los lineamientos generales de acción mediante diversos mecanismos. A pesar de ello, y con base en lo planteado con anterioridad, es igualmente posible identificar de manera precisa algunos argumentos que permiten visualizar que estas organizaciones tienen cierta autonomía y, por ende, la capacidad de ejercer poder sobre los Estados y otros actores. En tal sentido, es posible plantear los siguientes argumentos a favor de la autonomía —al menos relativa- de las OI:

Jurídica y formalmente, las OI tienen personalidad jurídica propia, la cual puede ser expresa o tácita, y deducible de cierto tipo de mecanismos o funciones. Esto significa que son diferentes de sus integrantes y, por tanto, en cierta forma interactúan con ellos. Pero adicionalmente, si bien las organizaciones actúan con base en atribuciones de los Estados, cuentan con poderes implícitos e inherentes que se escapan de uno u otro modo de su control y que en la práctica han permitido un accionar propio. Este sería el caso, por ejemplo, del Banco Mundial, entidad que se ha venido 
introduciendo en el tema de los derechos humanos y el Estado de derecho a través de la reinterpretación interna de su mandato (Burgos, 2009).

El manejo de información especializada ha permitido que ciertas OI hayan logrado una autoridad propia en algunos temas, de manera tal que sus estudios, recomendaciones e incluso presiones sobre ciertos Estados se toman en cuenta en forma generalizada. Como ya se ha indicado, si bien el $\mathrm{BM}$ y el FMI se ven regularmente como meras plataformas de los intereses de Estados Unidos, las críticas de sectores estatales de dicho país a este y otros organismos como la ONU son permanentes, en tanto que sus estudios y recomendaciones se valoran en un sentido u otro en el marco de las luchas de poder dentro de los países. Lo que quiere plantearse por esta vía es que la especialización técnica de ciertos organismos les da un margen de autonomía relativo y analizable para cada caso y, por consiguiente, con la capacidad de ser un actor frente a ellos (Barnett y Duvall, 2004).

El ethos burocrático, ligado con el sentido de pertenencia a la organización y la defensa de su carácter independiente y especializado. Así, de la manera como Theda Skocpol (2007) lo ha planteado para entender el funcionamiento de los Estados, toda organización genera por intermedio de sus integrantes un ámbito de pertenencia y de sentido unido a sus objetivos, misión, tradiciones e historia, lo cual les da un nivel de acción propio y al margen de los actores de poder que se ubican por encima de ellos. Es así como se viene planteando la existencia de burocracias internacionales que van construyendo un sentido de vinculación a los correspondientes organismos, al interpretar sus mandatos abiertos y defender su carácter apolítico y técnico. Por esta vía, ciertas Ol logran ser actores por sí mismos, en cuanto tienen funcionarios cohesionados y con altas capacidades que les dan cierta autonomía frente a los Estados (Reinalda y Verbeek, 2003; Groenler, 2009).

La dinámica burocrática se ha estudiado igualmente a la luz de las llamadas normas informales de las Ol. Esto significa que una cosa es su análisis a partir del estudio de sus marcos formales, expresados en tratados y textos escritos, en los que se establecen funciones y objetivos, y otra parcialmente distinta es el funcionamiento de acuerdo con ciertos patrones de la OI, donde existen tradiciones, cambios de metas, luchas de poder no sujetas a textos escritos, pero sí a ciertas dinámicas identificables (Arato, 2013; Stone, 2013). 
Para las organizaciones internacionales, esto significa que deben ser reconocidas por la comunidad internacional como actores y que en esa comunidad sus decisiones deben tener algún impacto. Los abogados internacionales podrían ver esta personalidad como un producto de la construcción legal de la organización mediante su tratado, mientras que los científicos políticos y los sociólogos probablemente la vean como un producto de un proceso social de institucionalización (Hurd, 2011, p. 28. Traducción propia).

Tomando en cuenta entonces que aun en el marco de las diferencias analíticas sobre la racionalidad del accionar de las Ol es posible identificar la idea relativamente común de que son algo que va más allá de la mera voluntad e interés de los Estados y que, por tanto, tienen ámbitos propios de acción, cabe preguntarse cuáles pueden considerarse sus recursos de poder para actuar sobre los Estados y conseguir algún nivel de obediencia.

\section{Las organizaciones internacionales y sus recursos de poder}

Dentro de la Ciencia Política el poder ha tendido a definirse desde los términos de su detentación, la lógica del acceso y tenencia de ciertos [158] recursos, y finalmente como un tipo especial de relación social. Desde una perspectiva del poder como detentación, las OI cuentan con las facultades delegadas por los Estados de algún modo y por ello tienden a considerarse como meros mandatarios, fundados en una legitimidad de fines y medios. Por su parte, desde la lógica clásica de los recursos de poder —el conocimiento, el capital y la coerción-, pueden estimarse como detentadoras variables de las dos primeras, y la fuerza es inicialmente un terreno, ante todo, estatal (Keohane, 1993). ${ }^{1}$

Estos dos planteamientos sobre el poder se han cuestionado por varias vías y de manera general. En primer lugar, sostener que el poder es algo que se detenta, mediante el acceso a ciertos recursos o delegaciones, corporiza el poder, en cierto modo lo materializa, cosa que no es empíricamente verificable en forma plena, y además transmite que si algunos tienen el poder

\footnotetext{
${ }^{1}$ El acceso al conocimiento y a la información da lugar al poder cultural o ideológico, en tanto que la detentación de recursos económicos, los cuales son históricamente variables y hoy están monetizados o expresados en recursos naturales, tecnología, entre otros, genera los ámbitos propios del poder económico. Por su parte, la fuerza organizada en forma permanente es el recurso propio del poder político como tal (Bobbio, 1989).
} 
como algo corpóreo, otros no lo tienen o lo tienen en menor proporción o cantidad, cosa que tampoco es verificable; adicionalmente, hay aquí una visión unilateral y superficial del poder en cuanto se mira desde quien lo ejerce y no desde quien dice o no obedecer. Dicha unilateralidad, por lo demás, transmite la muy limitada idea de que solo por tener poder o recursos de poder se obtiene la obediencia, cuando es fácil determinar que no siempre el acceso a ciertos recursos o competencias conduce a ser obedecido, sino que además —y más claro aún— desconoce que no siempre se obedece, debido a que hay resistencias y cuestionamientos a quienes supuestamente tienen el poder. Según estos enfoques, el poder debe conllevar el logro de la obediencia en las relaciones sociales para existir y, como se acaba de plantear, no por tener un supuesto poder se es obedecido, sino que, por el contrario, se puede ser resistido (Burgos, 2018).

Otra entrada aplicable afirma que las Ol se encuentran en una posición estratégica frente a otros actores en el marco de relaciones cambiantes, donde se emplean diversos recursos: «El poder no es una institución, no es una estructura ni una fuerza de la que dispondrían algunos: es el nombre que se le da a una situación estratégica compleja en una sociedad dada» (Montbrun, 2010, p. 1). En otras palabras, el poder es ante todo un tipo especial de relación social, que si bien se ha definido en términos negativos, es decir, afirmando que es algo que no se puede detentar, implica tomar en cuenta diversos elementos analíticos: primero, si el poder es algo relacional, tiene que ver con las interacciones sociales entre seres humanos o sujetos creados por estos - Estados, empresas y $\mathrm{Ol}$ - , aspecto en el que se puede obedecer o no cierto imperativo, mecanismo o mensaje. Dado que estamos en presencia de relaciones, estas pueden ocurrir en el sentido de obedecer o desobedecer de manera fluida y cambiante, según ámbitos diversos de poder (Burgos, 2018).

Esto implica, en segunda instancia, admitir que nos encontramos en presencia de múltiples relaciones de poder a escala social, sean estos la familia, la empresa y las organizaciones, dentro y fuera del Estado. El poder está entonces regado en las relaciones sociales, por lo que hay un variado tipo de actores de poder, de modo tal que no es fácil afirmar su centralización en algún sitio, como suele ocurrir con el Estado (Burgos, 2018).

En tercer lugar, parafraseando a Max Weber: «todas las cualidades imaginables de un hombre y toda suerte de constelaciones posibles pueden 
colocar a alguien en la posición de imponer su voluntad en una situación dada» (Tapia, 1999, p. 10). Así, el sexo, la altura, los uniformes, la belleza y la capacidad retórica, entre otros, son recursos que en determinado momento nos permiten ejercer poder sobre otros, de manera tal que, adicionalmente, estos son históricos, es decir, cambiantes en el tiempo (Burgos, 2018).

En cuarto lugar, vale la pena reiterar que ciertos recursos son considerados más idóneos para poder ejercer el poder en cuanto son escasos y valiosos para un colectivo, tales como el conocimiento, la información, el dinero o la fuerza. Finalmente, las relaciones de poder tienden a ser asimétricas, dado que, al menos de modo temporal, nos encontramos en la situación de decisiones que pueden ser imperativas o manipuladoras para obtener obediencia (Tapia, 1999). Aquí parece claro que hay que destacar la relación entre el poder como el acceso a ciertos recursos y el ejercicio de este. En efecto, no son los recursos en sí los que generan obediencia, sino su uso, el cual es dinámico y cambiante. En tal sentido, lo principal es el ejercicio:

En suma, el poder es un concepto relacional. Esta afirmación hay que entenderla en dos sentidos. El poder es, por una parte, una relación social y no algo que se posea, y por otra, las entidades que se utilizan para establecer dicho tipo de relaciones adquieren el estatus de recurso en la medida en que los otros la valoran como tal. En otras palabras, para que una entidad sea considerada como recurso de poder, debe ser valorada tanto por el actor «a» como por el actor «b». Esto último implica que para que una entidad sea concebida como recurso de poder depende de una valoración recíproca y no de una estimación unilateral (Tapia, 1999, p. 4).

Reconocer que el poder no es algo que se detenta sino que se ejerce permite tener en cuenta que en el interior de determinados actores de poder hay luchas en torno a este. En efecto, ya desde el liberalismo clásico se plantea que para evitar abusos en el poder del Estado, por ejemplo, este debe dividirse en su ejercicio a través del clásico tripartito ejecutivo, legislativo y judicial, lo cual lleva aparejados pesos y contrapesos entre ellos, es decir, potenciales y reales tensiones y conflictos a causa de los controles mutuos existentes. Con todo, aparte de esta clásica división que conlleva tensiones en el interior del poder del Estado, hay otra clase de tensiones y luchas de poder, las cuales se dan entre organismos de control respecto a otros ámbitos estatales, entre las entidades descentralizadas y los organismos centrales, y entre organizaciones de las que se han apropiado sectores de 
partidos políticos, universidades, representantes de regiones, corporaciones burocráticas sindicales o no, así como actores ilegales que han cooptado al Estado. Debido a la existencia de varios actores de poder, el Estado se muestra en su interior como un campo de lucha antes que como un actor homogéneo (Burgos, 2018).

Aplicado al caso de ciertas Ol —como el Banco Mundial-, históricamente se han presenciado los debates internos entre la noción de desarrollo iniciada por los ingenieros y agrónomos, y la que luego propusieron los economistas. Más recientemente están las luchas entre estos últimos y los abogados sobre, por ejemplo, la legitimidad y el alcance de involucrarse en una agenda de derechos humanos como conjunto de obligaciones jurídicas antes que como resultado del proceso de desarrollo, tema que se abordará en la tercera parte de este texto (Sarfaty, 2012; Elliott, 2015).

En suma, las Ol son actores de poder en cuanto forman parte de relaciones estratégicas, en las que hacen uso de determinados recursos que pretenden y logran, hasta cierto punto, la obediencia de los Estados y otros actores de poder; por tanto, estas organizaciones entran en juegos y luchas de poder a partir del uso variado y cambiante de dichos recursos, y no meramente porque posean una parcela de poder en sí mismas, según la idea clásica de que el poder se tiene o no.

A partir de los anteriores referentes y de otros insumos, a continuación se planteará que los recursos de poder de las OI se pueden identificar como normativos, informativos, coercitivos y simbólicos, los cuales no siempre se dan en todas estas, pero su enunciación analítica puede ser útil para verificar su existencia o no, lo que permite determinar su peso específico como un actor de poder.

\subsection{Poderes normativos}

Aquí se habla de aquellas atribuciones de las OI que remiten a la producción de normas de distinto tipo y que tienen un carácter general, normalmente respecto a los Estados integrantes y autorizadas por ellos de una u otra manera. Este poder normativo se expresa a través del mecanismo de las resoluciones no vinculantes o sus equivalentes, emitidas por diversos órganos, además de la verificación de ciertas normas internas como parte del proceso de adhesión a algunas de ellas. Igualmente, pueden encontrarse 
la codificación de la costumbre existente en cierta área o el monitoreo respecto del cumplimiento de las normas, aun cuando estas no sean del todo vinculantes, como se ha afirmado (Gutiérrez, 2014).

Si bien no todas las OI tienen atribuciones normativas expresas, aquellas que las tienen pueden producir resoluciones a título de recomendaciones, es decir, no vinculantes, dirigidas fundamentalmente a sus Estados miembros, las cuales les permiten desarrollar su objeto y fines. Una de sus características principales es su carácter formal (Monroy, 2000). Es así como, por ejemplo, una parte de las resoluciones del Consejo de Seguridad de la ONU, de la Asamblea General de este organismo o los equivalentes de directrices del Banco Mundial o del FMI, entre otros, no son en estricto sentido jurídicamente obligatorias o exigibles a los Estados (Wouters y De Man, 2011).

Lo anteriormente admite excepciones en sentido formal, pero igualmente en la práctica. En cuanto a lo primero, existen - para ciertos casos-ámbitos normativos de decisión vinculante, como ciertas resoluciones del Consejo de Seguridad de la ONU respecto del capítulo vII de la Carta de la ONU; resoluciones de la Organización Internacional del Trabajo (OIT) en relación con los compromisos previstos en ciertos tratados; decisiones

[162] vinculantes de ciertos órganos de la OMC o de la Unión Europea. Por otra parte, es posible que resoluciones de ciertas OI terminen convirtiéndose en costumbre internacional, es decir, en norma vinculante del derecho internacional público, como ya ha ocurrido con ciertas decisiones de la Asamblea General de la ONU. Existe un poder de legislar en el Consejo de Seguridad cuando, por ejemplo, «adopta resoluciones por las que requiere a los Estados que congelen fondos y activos de individuos sospechosos o implicados en actividades» (Gordillo, 2012, p. 206).

Ahora bien, reconocer la regla de la no obligatoriedad jurídica de las normas producidas por ciertas OI no significa que en la práctica no tengan fuerza y eficacia respecto de espacios de acción de los Estados. Con la expresión «derecho blando», al que se ha ligado una parte de la producción normativa de dichas organizaciones, algunos Estados han tendido a obedecer de manera desigual las normas emitidas por estas, en la medida en que su adopción está asociada a beneficios reputacionales, los cuales en algunos casos están vinculados igualmente a luchas de poder internas (Daugirdas, 2014). Un caso prototípico al respecto es la Organización para la Cooperación 
y el Desarrollo Económicos (OCDE), cuyas resoluciones y mecanismos de monitoreo producen decisiones en algunos casos no vinculantes, pero que son acatadas por los Estados miembros y en algunos casos sirven de referente para otros que no lo son, en razón de los beneficios reputacionales que entraña para ciertos círculos de poder comprobar la adopción, al menos formal, de ciertas decisiones que conllevan la homogeneización de políticas. Aún más, actores de la sociedad civil de ciertos países se apropian de resoluciones de la Asamblea General de la ONU y las agencian con el fin de presionar su adopción como normas nacionales vinculantes por los Estados donde se encuentran asentadas.

Hay una situación intermedia en el caso del Banco Mundial a partir de las políticas de salvaguardia relacionadas con los derechos humanos. El $\mathrm{BM}$ ha venido generando decisiones en las que interpreta y apropia ad hoc normas en materia de derechos humanos, que espera que se apliquen en los países miembros, asociadas a sus préstamos. Por lo demás, se convierten en referentes para otros organismos internacionales y para los mismos países en cuanto guías del comportamiento. Esta mezcla entre derecho blando que genera precedentes, atado al poder económico de ciertos bancos multilaterales, y que a su turno es una referencia para otros actores, lleva a sostener que «[...] las IFI [instituciones financieras internacionales] también son cada vez más relevantes como creadores de derecho internacional, al menos en el sentido de establecer precedentes (o ejemplos) que puedan ser utilizados por otros en el desarrollo del derecho internacional» (Bradlow y Hunter, 2010, p. 27).

En suma, el poder normativo de las OI hace referencia a las atribuciones que tienen para generar modelos de comportamiento vía resoluciones y directrices que, si bien no siempre son formalmente vinculantes, fácticamente alcanzan algún nivel de eficacia de manera desigual. Este sería el producto del convencimiento de los Estados que respaldaron su emisión por intermedio de sus representantes, pero también del análisis sobre los costos reputacionales que significa no hacerlo. A su vez, estos se desdoblarían en la generación de una imagen de país cumplidor de las normas internacionales o, contrario sensu, de medir las consecuencias de ser incumplidor a este nivel. A partir de dichos elementos, actores internos presionan a sus gobiernos e instituciones para que adopten internamente la normativa que se considera adecuada en algún momento, solapándose con el poder simbólico de los organismos. 


\subsection{Poder informativo}

La información es un recurso de poder clásico que hace referencia a la forma como se utiliza para obtener obediencia o, en otros términos, el acceso a ciertos datos o conocimiento decantado según diversas metodologías y fuentes que permiten influenciar efectivamente el comportamiento de otros.

Las organizaciones internacionales generan diverso tipo de información y de conocimiento, el cual se puede agrupar en varias categorías: por un lado, realizan trabajos generalmente comparados y con series de tiempo largas, por lo regular, sobre los temas a los cuales están dedicadas. Entre estos cabe destacar los informes periódicos en los que se analizan el comportamiento de los países a la luz de ciertas variables, tales como el Doing Business o el Rule of Law Index del Banco Mundial, o el Índice de Desarrollo Humano del Programa de las Naciones Unidas para el Desarrollo (PNUD), adscrito a la ONU, y que establecen categorizaciones (Barnett y Duvall, 2004, p. 172).

Por otra parte, en el seno de ciertas Ol se producen documentos dirigidos a replantear o resignificar ciertas categorías clásicas, como el caso de las transformaciones de la idea de soberanía a la luz de la intervención

[164 ] humanitaria hecha por la ONU o de los conceptos de gobernanza y desarrollo expresados por el FMI y el Banco Mundial. Igualmente, ligados a los anteriores, se producen documentos de recomendaciones dirigidos a los Estados. Otra de las formas en que tales datos aparecen es mediante la recolección y socialización de las, hoy conocidas, mejores prácticas (Burgos, 2009).

Esta batería amplia y compleja de información y conocimiento, la cual tiende a estar sustentada en modelación matemática y estadística, respaldada por expertos en las diversas áreas, generalmente reclutados en universidades de élite, tiene como propósito influir en la agenda interna de generación de políticas (Barnett y Duvall, 2004, p. 177). En efecto, desde poner el tema en la discusión nacional a partir de la difusión de un estudio fundado en una $\mathrm{OI}$, hasta proyectar su abordaje y adoptar las recomendaciones directas o indirectas de estos, son impactos variados que surgen de los recursos informativos de poder usados por estos sujetos. Esto generalmente ha ocurrido por medio de la adopción de dichos reportes por sectores de las élites que toman decisiones, las cuales vehiculan sus contenidos a escala nacional y les brindan y reconocen legitimidad a esta clase de trabajos. En otros términos, dado el poder simbólico, los estudios e informes de ciertas OI se consideran 
idóneos según ciertas élites que los «nacionalizan» y se convierten en agenda nacional. Esto ocurre tanto para sectores de gobierno que utilizan los informes del BM y el FMI, como para los grupos expertos en la defensa de los derechos humanos que hacen lo propio con documentos de las Naciones Unidas o el Fondo de Desarrollo de las Naciones Unidas para la Mujer (Unifem), por ejemplo. ${ }^{2}$

¿Cómo expresa y capta una organización el proceso de toma de decisiones y planificación de un Estado? ¿Cómo visualiza su producto final? Las respuestas a estas preguntas varían según las organizaciones internacionales. Por ejemplo, las empresas multinacionales y las organizaciones internacionales atentas a los beneficios de los miembros (como el ICC) pueden perseguir numerosas estrategias de producción, tales como 1) realizar o financiar estudios que respalden una conclusión o enfoque particular; 2) desarrollar puntos de discusión y declaraciones de posición para hacer cabildeo a los Estados, y 3) crear alianzas informales o formales con otras organizaciones que comparten una disposición similar sobre el tema en particular (Ring, 2009, p. 659).

En resumen, la producción de información y conocimiento especializado, por lo general, por largas series de tiempo, de manera comparada, con metodologías altamente cuantitativas y presentadas a través de informes, documentos analíticos, escalafones, mejores prácticas, entre otros, es lo que se identifica como una socialización coercitiva o un entramado de poder que impacta de manera blanda en el accionar de ciertos Estados (Barnett y Duvall, 2004, p. 179). De modo inicialmente «libre», ciertas élites de Estado se adaptan a los análisis y reproducen las recomendaciones de algunos organismos internacionales, legitiman sus políticas en la idoneidad de dichos estudios y, en la práctica, terminan afrontando las disputas de poder en torno de ciertas decisiones a partir de los estudios de tales organismos. Así, la información y el conocimiento generado sistemáticamente por ciertas OI crean y organizan, de algún modo, la realidad al establecer cuáles son los problemas y cuáles las soluciones.

\footnotetext{
${ }^{2}$ Uno de los hechos más recientes al respecto tuvo lugar a propósito de las declaraciones del hoy ex economista jefe del Banco Mundial, Paul Romer, quien admitió que los datos del Doing Business se orientaron para desacreditar al gobierno de la presidenta Michelle Bachelet, lo cual tuvo un alto impacto dentro de los actores de poder en Chile; además, no se descarta que haya influido en los resultados de las elecciones a la Presidencia que ganó Sebastián Piñera (Montes, 2018, enero 15).
} 


\subsection{Poder coercitivo}

La obediencia a cierto marco normativo es el producto de alguna forma de imposición unilateral. En sentido general, se puede afirmar que las OI carecen de poderes coercitivos, de manera similar a como los Estados lo tienen internamente, los cuales, por lo demás, se han cuidado bastante en no delegar tales prerrogativas a entramados internacionales. Internacionalmente, la fuerza como recurso de poder es el terreno de órganos como el Consejo de Seguridad de las Naciones Unidas y la Organización del Tratado del Atlántico Norte (OTAN), en tanto que el recurso económico hace referencia al mecanismo de préstamos condicionados provenientes de los llamados bancos multilaterales de desarrollo, pero igualmente incluye el uso de sanciones económicas por parte de la ONU respecto de Estados que violan ciertas normas del derecho internacional. Así, lo que se denomina sanciones político-administrativas corresponde a medidas como las de la OEA, la UE o el Mercosur respecto de Estados que desconocen normas en materia de democracia y derechos humanos, y como consecuencia pierden ciertos derechos dentro de la OI (Simmons y Martin, 2002, p. 329).

En lo relativo al comportamiento del recurso de la fuerza armada [166] por la vía fundamentalmente de decisiones del Consejo de Seguridad de la ONU, debe afirmarse que es excepcional y no siempre eficaz. En efecto, luego de la caída del muro de Berlín, el mencionado órgano ha autorizado el empleo de la fuerza en casos como los de Kuwait, Somalia, la antigua Yugoslavia, entre otros, pero como se sabe, las que hacen uso de esta son las potencias mundiales, encabezadas generalmente por Estados Unidos y sus llamados aliados (Thompson, 2015). En otras palabras, no solo el uso de la fuerza internacional es excepcional, sino que cuando ocurre no es la misma ONU la que lo planea y dirige, sino algunos Estados integrantes de esta organización, o en el mejor —o peor- de los casos la OTAN, como ocurrió en la acción militar contra Libia en cuanto parte de las dinámicas de intervención humanitaria. Esta doble excepcionalidad impacta de una u otra manera en su eficacia, sobre todo si se toma en cuenta que, si bien el Consejo de Seguridad aprueba ex ante o ex post el uso de la fuerza, no es el responsable de su aplicación y de su monitoreo. Incluso en aquellos casos en que ciertos Estados miembros han utilizado la coerción armada internacional en nombre de la ONU esta ha tenido un impacto inmediato para conjurar ciertas amenazas a la paz y la seguridad internacionales, pero a largo plazo no ha sido la forma más idónea para afrontar sus causas, como es fácil de 
verificar respecto de Irak y Afganistán, donde la acción armada internacional estaría detrás del surgimiento del Estado islámico y del fortalecimiento actual de los talibanes (Thompson, 2015).

Otro de los recursos que permiten establecer algún tipo de coerción desde las OI tiene que ver con la facultad de prestar dinero de manera condicionada, algo que hacen generalmente los llamados bancos de desarrollo o los entramados interestatales dirigidos a atajar crisis financieras. Se hace referencia al Banco Internacional de Reconstrucción y Fomento (BIRF), a los bancos regionales para América, África y el este de Europa, al FMI y a sus homólogos regionales como el Fondo Monetario de Europa (Simmons y Martin, 2002, p. 334).

Al ubicar la condicionalidad se habla de que el otorgamiento de ciertos préstamos esté sujeto al cumplimiento de requisitos que se pueden clasificar de dos maneras: a) requerimientos previos y durante el desarrollo de un proyecto o programa atado a un préstamo, los cuales de alguna manera enmarcan tanto su aprobación como la entrega parcial de ciertos desembolsos; b) existencia de un benchmarking respecto de ciertas políticas o, dicho en otros términos, referentes de acción que no afectan los desembolsos pero son una forma de «poder blando» en relación con los países y sus políticas en ciertos temas (World Bank, 2005, p. 43). ${ }^{3}$

Los préstamos aprobados por estos organismos están enmarcados en el amplio terreno de la búsqueda del desarrollo, la lucha contra la pobreza y el enfrentamiento de crisis financieras, según los ámbitos de acción de cada uno de ellos. No obstante, con tales rótulos aparece un ingente grupo de temas fundados en la tesis de su utilidad para lograr esos macroobjetivos, de modo tal que los préstamos condicionados incluyen referencias sobre la reforma del Estado y del aparato de justicia, la privatización de empresas públicas y la aplicación de políticas educativas, ambientales y en materia de género (World Bank, 2005, p. 23).

A pesar de su ampliación temática, la dimensión de la condicionalidad de los préstamos presenta ciertas características recientes. Por un lado, los

\footnotetext{
${ }^{3}$ Aquí se puede ver cierto solapamiento con lo que aquí se denomina poder de la información o el conocimiento y el carácter blando pero eficaz de la normativa producida por ciertas OI. Esto deja en claro que la propuesta presentada no significa la separación plena de los recursos de poder sino, según el caso, su interacción.
} 
fondos de ciertos organismos como el BM y el BID no son ilimitados, lo cual conlleva a que los recursos se dividan en programas y proyectos que no cubren a la mayoría de los Estados del mundo y que, en cierta forma, restringen su alcance a la hora de obtener ajustes internos de algún tipo. Es claro que en la actualidad la financiación pública vía el endeudamiento externo es un terreno de los actores financieros privados o estatales de carácter global, al menos para los países de desarrollo medio hacia arriba, financiación que no tiende a tener el tipo de condicionalidad astringente generalmente asociada a los bancos de desarrollo (Hernández, 2017).

Sin embargo, esta restricción al alcance de los préstamos condicionados es morigerada, si se considera que los países más afectados por la condicionalidad de este tipo de OI son aquellos que no pueden acceder a financiación internacional vía los mercados privados. Esto significa que el poder coercitivo de la condicionalidad afecta a Estados que tienen menos capacidad de resistirla de alguna manera dadas sus pocas opciones para hallar otras formas de financiamiento (Hernández, 2017). ${ }^{4}$

La condicionalidad es una especie de coerción en al menos dos sentidos. Si bien en el aspecto formal sus cláusulas son parte de un acuerdo entre el [168 ] Estado y la Ol correspondiente, en la práctica la aprobación de un préstamo, del cual depende la confianza respecto de ciertos actores globales, y el flujo para hacer ciertos gastos de Estado son una clara presión frente a algunas autoridades públicas a favor de condiciones cuyo contenido está delineado, en buena parte, por los bancos antes que por los Estados. Dicha situación se hace más fuerte si se considera que las condiciones terminan alcanzando áreas no estrictamente macroeconómicas, como los casos de la reforma del Estado o del sector de la justicia en ciertos países (Pender, 2001). ${ }^{5}$

Ahora bien, el cumplimiento de las condiciones por parte de los Estados, en cuanto expresión del alcance de la coerción, es algo ambiguo. En efecto, en varios estudios se ha constatado que aquellos no siempre cumplen las condiciones según lo esperado y que a pesar de esto continúan recibiendo los desembolsos. Esta situación varía en cada país debido a que dicha tolerancia

\footnotetext{
${ }^{4}$ Haití es uno de los casos más recientes, donde por exigencia del FMI se trataron de eliminar ciertos subsidios a algunos combustibles, lo cual degeneró en una reacción social que al final terminó con la reversión de las medidas y la salida del primer ministro del Ejecutivo.

${ }^{5} \mathrm{La}$ condicionalidad es el producto de la negociación de los Estados y las OI con la intervención de ciertas potencias mundiales, las cuales sostienen la pretensión de algunos de estos últimos respecto de ciertas condiciones que van a ser impuestas por el financiador (Hernández, 2017).
} 
al incumplimiento depende de las relaciones que se tengan con ciertas potencias. En otros términos, élites nacionales, con el respaldo de Estados Unidos o Alemania dentro del Banco Mundial, por ejemplo, pueden sortear flexiblemente el cumplimiento de las condiciones, mientras que aquellos que no están en dicha situación lo encuentran más difícil (Hernández, 2017).

Así las cosas, la coerción armada o económica está presente en algunas Ol con un nivel de eficacia diferencial, ya sea porque la fuerza no siempre es ejercida por las organizaciones o porque las constricciones económicas tienen un margen para la desobediencia, según la relación del país con algunos de aquellos que son potencia y que están vinculados al organismo internacional en cuestión.

\subsection{Poder simbólico}

A partir de los trabajos de Pierre Bourdieu se logró determinar la existencia de un campo de poder llamado simbólico, caracterizado por tratar de entender cómo el conocimiento y el lenguaje son fundamentales en la legitimación de las luchas políticas. Dicho poder se expresa en la posibilidad retórica de convertir los intereses de un grupo o facción en lo que puede considerarse correcto, oficial, valioso u ortodoxo. Esto descansa en el papel que cumplen ciertos actores para establecer dichas verdades en cuanto se les reconoce autoridad para ello y en tal sentido son debidamente legitimados porque cuentan «con el suficiente reconocimiento como para imponérselo a los otros, y estructura visiones en una relación determinada con la realidad»; es el poder «de consagrar o revelar las cosas que ya existen» (Bourdieu, citado en Somohano, 2012). A diferencia de lo que más atrás se denominó poder del conocimiento, no se trata tan solo del manejo de cierta información sistematizada sino de la generación de referentes que organizan jerárquicamente el mundo y que son transmitidos por algunas instancias, como las OI, a las cuales se les reconoce la capacidad de decir lo que se juzga aceptable. El poder simbólico puede verse como un paso cualitativo, más allá de la mera instrumentalización de cierto conocimiento (Eagleton-Pierce, 2013).

En varios estudios se ha anotado que algunas organizaciones internacionales han acumulado un capital simbólico que les permite ejercer cierto poder al reconocerles y otorgarles la cualidad de estar por encima de las diferencias nacionales, de las tensiones entre grupos, y en tal sentido logran desempeñar un papel como mediadoras, generándose y asumiéndose 
una legitimidad para ello. Además, son promovidas y reconocidas como las que están autorizadas para decir lo correcto en ciertas situaciones. De hecho, ambas funciones están claramente interconectadas, aunque pueden diferenciarse en cuanto uno sería el rol de tercería asociable a diferencias internas intramitables y otro el rol de autoridades de ciertas «verdades», las cuales a veces facilitan ciertas tercerías (Hehir y Robinson, 2007).

El poder simbólico se genera únicamente cuando hay «una creencia en la legitimidad de las palabras y de la persona que las pronuncia, una creencia que las palabras mismas no pueden producir». Al estudiar la legitimación de ciertas formas de lenguaje se puede comprender mejor cómo solo los intereses políticos particulares, ideas y prácticas se reconocen como «aceptables», mientras que otros luchan para ser apreciados o incluso son «olvidados» (Eagleton-Pierce, 2013, p. 67).

La ONU es uno de los casos prototípicos de lo anterior. En efecto, en ámbitos de tensiones especialmente políticas o incluso relativas a la lucha contra la corrupción, instancias de este organismo han adquirido un rol como verificadoras de los términos de la desmovilización de las Fuerzas Armadas Revolucionarias de Colombia (FARC) en Colombia o como actores [170 ] protagónicos de la Comisión contra la Corrupción en Guatemala. A su vez, la ortodoxia general sobre qué se entiende por derechos humanos, qué alcances y limitaciones tiene, a quiénes compromete, entre otros, es una tarea en gran parte asumida y reconocida a las Naciones Unidas, entidad que, por lo demás, es objeto de crecientes demandas para que se involucre en nuevos temas al respecto, por ejemplo, el reconocimiento de los campesinos como un sujeto colectivo a título de pueblo o la enunciación del derecho a la paz. La OEA y la Unión de Naciones Suramericanas (Unasur), organizaciones políticas por definición, han cumplido roles similares, sobre todo en materia de tercería neutral (Ulloa y Guzmán, 2012; Richmond, 2004; Hudson y Taylor, 2010).

Desde un marco más ligado a la autoridad de decir lo aceptable y lo legítimo, el Banco Mundial, el FMI y la OCDE comparten, en cierta forma, el oligopolio de defender una clase de globalización económica basada en el papel del mercado y en las transformaciones del Estado en diversos sectores. Otro tanto sucede con la OMC, que sigue siendo el faro de lo que se considera el libre comercio. La OMS cumple este papel a la hora de decir qué es una enfermedad o no, al igual que cuáles son sus causas y eventuales respuestas (Peet, 2009; Chorev y Babb, 2009). De hecho, en algunos casos este poder 
simbólico es, en estricto sentido, lo más parecido a una marca. Es el caso de la Unesco con su ya famoso monopolio de establecer cuál es la noción y dónde estarían los sitios o dinámicas que pueden llamarse patrimonio arquitectónico, inmaterial o cultural de la humanidad (Black y Wall, 2001).

El poder simbólico de ciertas OI, sea por la vía de su aparente neutralidad, la generación de la ortodoxia y la idea misma de la marca-organización, lo han utilizado los actores de estas estructuras incluso para financiarse y ampliar su rol. En efecto, niveles institucionales de la ONU como el Programa de las Naciones Unidas para el Desarrollo (PNUD) o la oficina del Alto Comisionado de las Naciones Unidas para los Refugiados (Acnur) ejecutan política pública en ámbitos nacionales fundamentados en lo anterior, logrando mantener una burocracia y la vigencia de su papel, muy a pesar de que los Estados-nación en los órganos de decisión correspondientes internacionalmente se resisten cada vez más a darles ingentes recursos para su funcionamiento (Comotto, 2014).

\section{Conclusiones}

El objetivo analítico de este trabajo fue establecer las líneas gruesas de un marco teórico que permita su aplicación para casos concretos dentro del ámbito de las OI existentes y, por esta vía, permitir su refinamiento, cuestionamiento, complementación y mejoramiento. Por tanto, constituye una exploración teórica fundada en la sistematización crítica de parte de la bibliografía actual y pertinente al respecto.

El crecimiento de las OI después de la Segunda Guerra Mundial ha sido indudable, hasta el punto de que numéricamente superan a los Estados hoy existentes (Carlsnaes, Risse y Simmons, 2002). Además, se encargan de una creciente cantidad de tareas que en algunos casos nacen con ellas, pero en otros constituyen delegaciones recibidas de los Estados que las crean y, hasta cierta medida, las mantienen económicamente y las regentan.

El aumento de dichos entramados interestatales se ha asociado a distintas explicaciones, que van desde las que sostienen que su creación obedece a los intereses particulares a corto y largo plazo de los Estados, sobre todo los más fuertes, entre estos aquellos que aseguran que encarnan bienes públicos de carácter internacional que superan los intereses inmediatos de aquellos, hasta las que ven en las $\mathrm{OI}$ infraestructuras institucionales con un juego y autonomía propios. 
En este texto se adoptó la tesis de que las Ol tienen un nivel de autonomía variable respecto de los Estados que las crean, la cual depende del alcance de su personalidad jurídica, del ethos burocrático que construyen y del tipo de información y conocimiento que generan. Por lo anterior, se puede afirmar que estas son organizaciones de poder, es decir, con la capacidad de alcanzar algún nivel de obediencia respecto de los Estados y otros actores de poder social.

Después de haber ubicado a las OI como actores de poder, este artículo de reflexión se hizo la pregunta de cuáles son los recursos de poder que algunas de ellas utilizan para buscar o alcanzar algún nivel de obediencia por parte de los Estados. Esta inquietud se fundó en el paradigma teórico de que el poder hace referencia a una posición estratégica en el ámbito de las relaciones entre actores y que depende de la forma como se emplean ciertos recursos en torno de la obediencia colectiva; por lo tanto, se alinderó bajo un enfoque sociopolítico del poder.

Como respuesta, se sustentó que, dependiendo del caso, ciertas OI cuentan con recursos de poder normativo, ligados a constructos jurídicos que conforman una mezcla entre derecho duro y blando; informativos, relativos

[172] a la generación y uso de cierto tipo de información y conocimiento que permiten establecer una agenda y crear rangos a partir de los cuales se evalúa el accionar del Estado; coercitivos, correspondientes al uso de la fuerza y de la condicionalidad económica; y finalmente, los simbólicos, vinculados a la capacidad asumida y otorgada a ciertas OI de determinar qué es lo correcto, de cumplir el rol de actores neutrales y técnicos, conformando una especie de marca de lo deseable en ciertas áreas de la política pública.

Estos recursos no existen en todas las OI, motivo por el cual uno de los retos que abren esta propuesta analítica es visualizar cuándo y cómo se producen o no en casos concretos. Por otra parte, hay que tomar en cuenta que los mencionados recursos pueden operar de manera simultánea - y en algún caso solapada-, lo que no significa desconocer las diferencias en su aporte al estudio concreto de cada uno.

\section{Referencias bibliográficas}

1. Arato, Julian. (2013). Treaty Interpretation and Constitutional Transformation: Informal Change in International Organizations. Yale Journal of International Law, 38 (2), pp. 289-312. 
2. Barnett, Michael \& Duvall, Raymond (eds.). (2004). Power in Global Governance. Cambridge: Cambridge University. https://doi.org/10.1017/CBO9780511491207

3. Black, Hather \& Wall, Geoffrey. (2001). Global-Local Inter-Telationships in UNESCO World Heritage Sites. In: Teo, Peggy; Chang, T. C. \& Ho, K. C. Interconnected Worlds. Tourism in Southeast Asia (pp. 121-136). Amsterdam: Pergamon.

4. Bobbio, Norberto. (1989). Estado, gobierno y sociedad. México, D. F.: FCE.

5. Botero Marino, Catalina. (2016, julio 7). Whistleblowers. Ámbito Jurídico. Recuperado de https://www.ambitojuridico.com/noticias/columnista-impreso/ constitucional-y-derechos-humanos/whistleblowers

6. Bradlow, Daniel D. \& Hunter, David (eds.). (2010). International Financial Institutions and International Law. The Netherlands: Kluwer Law International.

7. Brodnig, Gernot. (2001). The World Bank and Human Rights: Mission Impossible? Carr Center for Human Rights Policy Working, Paper T-01-05. Retrieved from http://fletcher.tufts.edu/ /media/Fletcher/Microsites/praxis/xvii/Brodnig.pdf

8. Burgos Silva, Germán. (2009). Estado de derecho y globalización: el papel del Banco Mundial y las reformas institucionales en América Latina. Bogotá, D. C.: Universidad Nacional de Colombia.

9. Burgos Silva, Germán. (2018). El Estado moderno en cuanto «abstracción armada». Algunas reflexiones. Revista Republicana, 24. Recuperado de http://ojs. urepublicana.edu.co/index.php/revistarepublicana/article/view/451

10. Capella, Juan-Ramón. (1997). Fruta prohibida. Una aproximación históricoteorética al estudio del Derecho y del Estado. Madrid: Trotta.

11. Carlsnaes, Walter; Risse, Thomas \& Simmons, Beth A. (eds.) (2002). Handbook of International Relations. London: Sage.

12. Comotto, Sabrina. (2014). El rol de los órganos y organismos internacionales pertenecientes al sistema de Naciones Unidas en el inicio (1977) y consolidación (1987-1995) del proceso de descentralización de la política habitacional en la Argentina. (Tesis inédita de maestría). Flacso-Argentina, Buenos Aires.

13. Chorev, Nitsan \& Babb, Sara. (2009). The Crisis of Neoliberalism and the Future of International Institutions: A Comparison of the IMF and the WTO. Theory and Society, 38 (5), pp. 459-484. https://doi.org/10.1007/s11186-009-9093-5

14. Daugirdas, Kristina. (2014). Reputation and the Responsibility of International Organizations. European Journal of International Law, 25 (4), pp. 991-1018. https:// doi.org/10.1093/ejil/chu087

15. D'Aspremont, Jean. (2007). The Exercise of Powers by International Organizations Under the (Un)Due Control of Member States. London: Oxford University.

16. Dreyzin de Klor, Adriana \& Morales, Mariela. (2009). Ampliación del Mercosur: el caso Venezuela. Buenos Aires: Zavalia.

17. Eagleton-Pierce, Matthew. (2013). Symbolic Power in the World Trade Organization. London: Oxford University. 
18. Elliott, Leilani. (2015). How do Institutions Engage with the Idea of a Human Rights-Based Approach to Matters Involving Children? A Case Study of Unicef and the World Bank. (Doctoral Dissertation). Melbourne Law School, Melbourne.

19.Foot, Rosemary;MacFarlane, Neil\&Mastanduno,Michael.(2003).USHegemony and International Organizations: The United States and Multilateral Institutions. London: Oxford University. https://doi.org/10.1093/0199261431.001.0001

20. Gordillo Pérez, Luis I. (2012). Hacia una progresiva constitucionalización del poder sancionador del Consejo de Seguridad de la ONU. Estudios Constitucionales, 10 (1), pp. 201-244. https://doi.org/10.4067/S0718-52002012000100006

21. Groenler, Martijn. (2009). The Autonomy of European Union Agencies: A Comperative Study of Institutional Development. Amsterdam: Eburon Academic.

22. Gutiérrez-Solana, Ander. (2014). La legitimidad social de las organizaciones internacionales. Madrid: Marcial Pons.

23. Hehir, Aidan \& Robinson, Neil (eds.) (2007). State-Building: Theory and Practice. Nueva York: Routledge. https://doi.org/10.4324/9780203964712

24. Hernández, Diego. (2017). Are «New» Donors Challenging World Bank Conditionality? World Development, 96, pp. 529-549. https://doi.org/10.1016/j. worlddev.2017.03.035

25. Hudson, Andrew \& Taylor, Alexandra. (2010). The International Commission Against Impunity in Guatemala: A New Model for International Criminal Justice Mechanisms. Journal of International Criminal Justice, 8 (1), pp. 53-74. https://doi.

[174] org/10.1093/jicj/mqq003

26. Hurd, lan. (2011). Theorizing International Organizations. Choices and Methods in the Study of International Organizations. Journal of International Organizations Studies, 2 (2), pp. 7-22.

27. Keohane, Robert. (1993). Instituciones internacionales y poder estatal: ensayos sobre teoría de las relaciones internacionales. Buenos Aires: Grupo Editor Latinoamericano.

28. Ku, Julian. (2000). The Delegation of Federal Power to International Organizations: New Problems with Old Solutions. Minnesota Law Review, 85 (71), pp. 71-89.

29. Montbrun, Alberto. (2010). Notas para una revisión crítica del concepto de «poder». Polis, 9 (25), pp. 367-389. https://doi.org/10.4067/S071865682010000100022

30. Monroy, Marco Gerardo. (2000). Derecho Internacional Público. Bogotá, D. C.: Temis.

31. Montes, Rocío. (2018, enero 15). El Banco Mundial alteró la calificación de Chile durante el gobierno de Bachelet. El País. Recuperado de https://elpais.com/ economia/2018/01/14/actualidad/1515899491_574904.html

32. Negri, Antonio. (2005). Europa y el Imperio. Madrid: Akal. 
33. Pender, John. (2001). From «Structural Adjustment» to "Comprehensive Development Framework»: Conditionality Transformed? Third World Quarterly, 22 (3), pp. 397-411. https://doi.org/10.1080/01436590120061679

34. Peet, Richard. (2009). Unholy Trinity: The IMF, World Bank and WTO. London: Zed Books.

35. Querejazu Escobari, Amaya. (2013). Responsabilidad de las organizaciones internacionales por actos ultra vires. Revista de Derecho, 39, pp. 1-29.

36. Reinalda, Bob \& Verbeek, Bertjan (eds.). (2003). Autonomous Policy Making by International Organizations. London: Routledge.

37. Richmond, Olivier. (2004). UN Peace Operations and the Dilemmas of the Peacebuilding Consensus. International Peacekeeping, 11 (1), pp. 83-101. https://doi. org/10.1080/1353331042000228403

38. Ridruejo, José. (2012). Curso de Derecho Internacional Público y de Organizaciones Internacionales. Madrid: Tecnos.

39. Ring, Diane. (2009). Who is Making International Tax Policy? International Organizations as Power Players in a High Stakes World. Fordham International Law Journal, 33 (3), pp. 649-675.

40. Sarfaty, Galit. (2012). Values in Translation. Human Rights and the Culture of the World Bank. Palo Alto: Stanford University.

41. Simmons, Beth A. \& Martin, Lisa. (2002). International Organizations and Institutions In: Carlsnaes, Walter; Risse, Thomas \& Simmons, Beth A. (eds.). Handbook of International Relations (pp. 192-211). London: Sage. https://doi. org/10.4135/9781848608290.n10

42. Skocpol, Theda. (2007). El Estado regresa al primer plano: estrategias de análisis en la investigación actual. En: Acuña, Carlos H. (comp.). Lecturas sobre el Estado y las políticas públicas: retomando el debate de ayer para fortalecer el actual (pp. 169-202). Buenos Aires: Jefatura de Gabinete de Ministros de la Nación.

43. Somohano Fernández, Abel. (2012). El concepto de poder simbólico como recurso para comprender la dimensión política de la comunicación masiva: hacia una posible articulación entre las propuestas de Pierre Bourdieu y John B. Thompson. Mediaciones Sociales, 10, pp. 3-33.

44. Stone, Randall W. (2013). Informal Governance in International Organizations: Introduction to the Special Issue. The Review of International Organizations, 8 (2), pp. 121-143. https://doi.org/10.1007/s11558-013-9168-y

45. Tapia, Luis. (1999). La idea del poder. Documento de Discusión, 23. Instituto de Ciencia Política de la Universidad de Chile.

46. Thompson, Alexander. (2015). Channels of Power: The UN Security Council and US Statecraft in Iraq. Ithaca: Cornell University. https://doi. org/10.7591/9780801459375

47. Urueña, René. (2008). Derecho de las organizaciones internacionales. Bogotá, D. C.: Universidad de los Andes. 
48. Ulloa, Fernando \& Guzmán, Sandra. (2012). Proceso de paz en Colombia. Participación de actores internacionales. Bogotá, D, C.: Ecoe.

49. Wade, Robert. (2001). Showdown at the World Bank. New Left Review, 7, pp. 124-143.

50. Wade, Robert. (2002). US Hegemony and the World Bank: The Fight over People and Ideas. Review of International Political Economy, 9 (2), pp. 215-243. https://doi.org/10.1080/09692290110126092

51. Wilkinson, Rorden. (2013). The WTO: Crisis and the Governance of Global Trade. London: Routledge.

52. World Bank. (2005). Legal Aspects of Conditionality in Policy-Based Lending. Review of World Bank Conditionality. Retrieved from http://documents.worldbank. org/curated/en/228751468134390047/pdf/428690WPOCondi10Box327331B01PUB LIC1.pdf

53. Women UN. (2015). Progress of the World's Women 2015-2016: Transforming Economies, Realizing Rights. Retrieved from http://progress.unwomen. org/en/2015/pdf/UNW_progressreport.pdf

54. Wouters, Jan \& De Man, Philip. (2011). International organizations as lawmakers. In: Klabbers, Jan \& Wallendahl, Åsa (eds.). Research Handbook on the Law of International Organizations (pp. 190-224). Cheltenham: Edward Elgar. https://doi. org/10.4337/9780857931290.00015 